\title{
BMJ Open Psychometric evaluation of the Arthritis Self-Efficacy Scale in Chinese adults with rheumatic diseases: a cross- sectional study
}

\author{
Tzung-Yi Tsai (D) , ${ }^{1,2,3}$ Ming-Chi Lu (D) , ${ }^{4,5}$ Hanoch Livneh, ${ }^{6}$ Miao-Chiu Lin, ${ }^{7}$ \\ Ning-Sheng Lai, ${ }^{4,5}$ How-Ran Guo (D) 3,8,9
}

To cite: Tsai T-Y, Lu M-C, Livneh $\mathrm{H}$, et al. Psychometric evaluation of the Arthritis SelfEfficacy Scale in Chinese adults with rheumatic diseases: a cross-sectional study. BMJ Open 2021;11:e042014. doi:10.1136/ bmjopen-2020-042014

- Prepublication history and additional material for this paper are available online. To view these files, please visit the journal online (http://dx.doi. org/10.1136/bmjopen-2020042014).

Received 23 June 2020 Revised 18 December 2020 Accepted 07 January 2021

Check for updates

(C) Author(s) (or their employer(s)) 2021. Re-use permitted under CC BY-NC. No commercial re-use. See rights and permissions. Published by BMJ.

For numbered affiliations see end of article.

\section{Correspondence to}

Tzung-Yi Tsai;

dm732024@tzuchi.com.tw and

How-Ran Guo;

hrguo@mail.ncku.edu.tw

\section{ABSTRACT}

Objective The Arthritis Self-Efficacy Scale (ASES) was designed to assess the degree of self-efficacy among patients with arthritis. Though the original English version of this instrument has shown a high degree of reliability and validity, a Chinese version of this scale has yet to be validated. Therefore, the aim of this cross-sectional study was to evaluate the psychometric characteristics of the Chinese version of ASES (C-ASES) in a population of Chinese adults with rheumatic diseases (RDs).

Methods After completing backward translation and expert validity, a convenient sample of 258 qualified participants with RDs from a hospital in Taiwan were recruited to explore the content validity, concurrent validity, construct validity, internal consistency reliability and testretest reliability of C-ASES.

Results The C-ASES has demonstrated acceptable internal consistency and test-retest reliability, with a Cronbach $\alpha$ of 0.91 and intraclass correlation coefficient of 0.89 , respectively. Concurrent validity was acceptable, with significant correlation between the subscales of the C-ASES and perceived depressive symptoms, as measured by the Taiwanese Depression Questionnaire $(p<0.05)$. The exploratory factor analysis revealed a three-factor solution (physical function, experienced pain and other symptoms) corresponding to the structure of the original instrument, which accounted for $59.78 \%$ of the total variance.

Conclusion Empirical data support the assertion that CASES is a reliable and valid screening instrument to assess self-efficacy in Chinese-speaking patients with RDs. C-ASES may be useful as a reference guide in providing appropriate interventions for bolstering self-efficacy among Chinese-speaking patients with RDs.

\section{INTRODUCTION}

Rheumatic diseases (RDs) are common conditions that affect the joints or the tissues surrounding the joint and other connective tissue. According to the US Centers for Disease Control and Prevention, more than 50 million adults in the USA have physiciandiagnosed arthritis, accounting for nearly one-fifth of adults experiencing this disorder. ${ }^{1}$ The common symptoms of RDs include
Strengths and limitations of this study

- Disease-specific measures offer valuable insights into the impact of a disease on various aspects of a patient's life.

- The topic of self-efficacy (SE) in patients with chronic diseases especially rheumatic diseases (RDs) has attracted much attention because higher levels of SE have been found to be associated with better coping with the disease and its clinical manifestations.

- As of now, no study has yet examined English to Chinese translation of the Arthritis Self-Efficacy Scale (ASES) or its psychometric properties among Chinese adults with RDs.

- The results of our study show that the ASES has good psychometric properties when it is applied to patients with RDs.

- The geographical and sociocultural limitations of the sample may restrict generalisability of the findings.

swelling, stiffness and decreased range of motion, all of which may lead to permanent disability. The estimated direct medical cost in 2003 for US patients with arthritis or other rheumatic conditions was $\$ 80.8$ billion, with the total societal costs (the sum of direct costs and indirect costs) estimated at $\$ 128$ billion. ${ }^{2}$ Similarly, in Taiwan, the annual healthcare expenditure for RDs treatment has increased over time, from 5.8 billion New Taiwan Dollars (NTD) in 2016, 6.3 billion NTD in 2017 to 7.1 billion NTD in $2018 .^{3}$ In addition to the enormous economic costs of RDs, they may trigger other illnesses. Some studies indicate that RDs may be associated with increased risks of kidney dysfunction, psychiatric disorders and cardiovascular diseases. ${ }^{4-6}$ Accordingly, patients with RDs have a $20 \%$ greater risk of all-cause mortality compared with the general population. ${ }^{7}$ Given these daunting figures, optimum disease control is essential to prevent or delay the complications associated with RDs. 
As patients are responsible for monitoring their disease daily, it is crucial that they maintain sufficient knowledge, skills and positive attitudes towards arthritis. The concept of self-efficacy (SE) has attracted increasing attention in clinical practice. ${ }^{8-11}$ The term was coined by Albert Bandura, an early cognitive psychologist. Bandura defined SE as a belief in one's ability to succeed in specific situations, accomplish a task or achieve a certain goal. ${ }^{12}$ The robust relationship between SE and self-care behaviours has been established among patients with RDs. ${ }^{8}$ Using SE models in designing educational interventions can significantly enhance self-management routines, which in turn lessen the risk of poor clinical outcomes, and assist patients in coping successfully with the manifestations of arthritis. ${ }^{9} 13$ These findings further indicated the need to evaluate SE before implementing therapeutic interventions for patients with RDs.

Following a detailed literature review, we discovered that SE and its related measures have been widely used, particularly via the Arthritis Self-Efficacy Scale (ASES). ${ }^{914}$ This scale was created by Lorig and colleagues in the late 1980s. ${ }^{15}$ The full 20-item ASES consists of three subscales (namely, pain, physical function and other symptoms) and has been validated and widely applied in many countries to measure degree of SE, including Sweden, ${ }^{16}$ Norway, ${ }^{17}$ Iran $^{18}$ and Turkey. ${ }^{11}$ Although these studies provide important references for the local context, to the best of our knowledge, the ASES has not been verified for its psychometric characteristics in individuals with arthritis in Chinese. Given these issues, this study aimed to explore the validity and reliability of the Chinese version of ASES (C-ASES) in Chinese arthritis subjects.

\section{METHODS}

\section{Study design and participants}

This cross-sectional study adopted a convenience sampling approach to recruit people with RDs from outpatient departments at a teaching hospital in Taiwan during 2018. The main inclusion criteria were as follows: (a) having physician-diagnosed RDs, such as ankylosing spondylitis, rheumatoid arthritis, Sjogren's syndrome, gout or systemic lupus erythematosus, (b) being older than 20 years and willing to participate in the study and (c) being able to communicate in either Mandarin Chinese or Taiwanese. Individuals who were unable to care for themselves or had physician-diagnosed psychiatric disorders before entry were excluded.

\section{Sample size calculation}

The sample size needed for this study was determined by Gorsuch's suggestion, which indicated that a minimum subject-to-item ratio for conducting factor analysis should be at least 5:1. ${ }^{19}$ Accordingly, because 20 items comprised the ASES, a sample of at least 100 subjects was required for sound data analysis. During the study period, 258 participants were recruited for data analysis.

\section{Ethics}

All of the potential participants were informed of the research purposes, procedures and their rights, and were required to provide written consent before participation. Additionally, all participants were informed of their rights under the Declaration of Helsinki.

\section{Instruments}

Three measures were used to survey the enrolled patients: ASES, the Taiwanese Depression Questionnaire (TDQ) and a questionnaire requesting demographic variables and clinical characteristics.

SE, the main focus of this study, was assessed by ASES. This measure was developed by Lorig and colleagues to identify and quantify SE among individuals with RDs. ${ }^{15}$ It is composed of 20 items clustered into three subscales of physical function (9 items), other symptoms (6 items) and pain (5 items), all scored on a 10-point (10-100) scale, with higher scores indicating greater SE. The instrument had been shown to have adequate reliability and to be a sensitive measure of SE for individuals with RDs. ${ }^{9} 10161720$ After obtaining consent from the scale developer (Dr Lorig), we used the reverse-translation method to improve the cultural validity and ensure the usefulness of ASES. ${ }^{21}$

Initially, this scale was translated into Chinese by two independent translators of Taiwanese descent who were fluent in both the original and the target languages to complete the forward translation. Any inconsistent or ambiguous phrase was highlighted and later agreed on following a thorough discussion between the two translators. Following this step, an additional independent English native-speaking translator who had not seen the questionnaire previously was invited to carry out the backward translation. Subsequently, a panel comprised of the principal researcher and all translators held a discussion which aimed to determine whether the adopted words and items conveyed the same meaning and confirmed that the used equivalent expressions did not, indeed, alter the cultural meaning of the original wordings. Throughout the translation procedure, we kept close contact with Dr Lorig, via email, to ascertain semantic consistency between the original copy and the backtranslated English version. Following these translational steps, we finished the development of C-ASES (see online supplemental file).

The TDQ is a 20-item self-reported questionnaire developed by Lee and colleagues to assess level of depression. ${ }^{22}$ Each item is scored on a 0-3 level, with the total scores ranging between 0 and 54 . Higher scores indicate greater psychological distress. Presently, it has been shown to be a psychometrically sound measure when used for Taiwanese patients with chronic illness. ${ }^{23}{ }^{24}$ For example, after applying the Structured Clinical Interview of the DSMIII-R diagnostic criteria as the gold standard, the TDQ showed good concurrent validity. ${ }^{22}$ The area under the received operating characteristics curve of the TDQ was 0.92. In regard to its reliability, the TDQ had previously 
demonstrated good internal consistency among different groups of subjects, with Cronbach's $\alpha$ ranging from 0.89 to $0.92 .{ }^{22-24}$ Cronbach's $\alpha$ for the present data was 0.92 . In the current study, it was applied to examine the sametime validity value between TDQ and C-ASES, since less depressed individuals have been shown to have higher $\mathrm{SE}$ and maintain higher positive health behaviours. ${ }^{25}$ For this reason, a reversed relationship is assumed to exist between SE and depression.

Lastly, through the medical chart review, we obtained data on each respondent's demographic and clinical characteristics, including sex, age, marital status, education level and household status. The clinical characteristics studied included the duration of RDs and comorbidity (defined as having at least one of the following diseases for at least 6 months duration: diabetes, hypertension, heart disease, stroke and cancer).

\section{Data collection procedure}

Before enrolling in the study, all participants received detailed written and verbal information on the aims and protocol of the study and signed an informed consent form. Throughout the procedure of data collection, all questionnaires were returned with no identifying personal information and were marked only with an encryption code to facilitate data analysis. The encryption rules were available to the researchers only. To investigate the testretest reliability of C-ASES, we administered the C-ASES again after 2 weeks to a convenience sample of 100 from the original participants.

\section{Data analysis}

The obtained data were analysed using SPSS V.16.0 for Windows (SPSS, Chicago, Illinois, USA). The characteristics of subjects and the mean $\pm \mathrm{SD}$ values of the main study variables were analysed. The reliability of C-ASES was estimated by calculating the internal consistency and test-retest reliability. Internal consistency of the scale was established using standard statistical procedures described by Cronbach's $\alpha$, and a Cronbach's $\alpha$ higher than 0.70 was considered satisfactory. ${ }^{26}$ The test-retest reliability was assessed using the intraclass correlation coefficient (ICC) together with the Bland-Altman procedure. The latter approach is a graphical technique that assesses the agreement between two measurements. By plotting the differences against the mean between two measuring points, it can disclose the magnitude of disagreement, highlight the outliers and facilitate identification of trend. Two rules suggested by Altman and Bland were used to the assessment of comparability. ${ }^{27}$ One is to discern whether the mean of differences was significantly different from 0 . The other is to test the dependency between difference and average of the two measurements by fitting a linear regression line, and a significant change in the slope of the regression line indicates disagreement between the two measurements.

In terms of validity measures, we examined the scale's content validity, face validity, concurrent validity and construct validity. Regarding the content validity, several experts in related fields were invited to examine the overall appearance of the pre-final version of the scale. To further support the scale's content validity index (CVI), we asked the expert panel to evaluate each item of the instrument using a 4-point scale ( $1=$ not relevant, $2=$ somewhat relevant, $3=$ quite relevant and $4=$ highly relevant). A pilot study was then performed to test the face validity of the items, which involved 30 participants from the target population. As to the construct validity of C-ASES, it was assessed using factor analysis. The Kaiser-Meyer-Olkin (KMO) measure of sampling adequacy and Bartlett's test of sphericity were used to determine whether the data were appropriate for factor analysis. Theoretically, a value of KMO below 0.50 means samples are not sufficient for factor analysis, whereas values above 0.90 are more than sufficient for factor analysis. ${ }^{19}{ }^{28}$ In order to determine the factorial structure of the scale, the principal components analysis was used to extract the factors, and varimax rotation was used to obtain the most meaningful original factor structure of the C-ASES. Regarding the concurrent validity, it was determined through the assessment of relation between C-ASES and TDQ by the Pearson correlation coefficient.

\section{Patient and public involvement}

Our participants took part in a pre-survey and a formal survey following the completion of the scale. Participation was voluntary, and no incentives were provided for participation. Participants were not directly involved in the design or development of research questions. The results of the study were not provided to individual participants.

\section{RESULTS}

\section{Sample characteristics}

Table 1 shows the distribution of demographic data and clinical characteristics among the enrollees. During the recruitment period, 258 patients with RDs were enrolled. The mean age of participants was 57.1 years $( \pm 14.1$ years $)$, and the majority were women $(74.4 \%)$, married $(84.5 \%)$ and had a high level of education $(51.2 \%)$, as well as a monthly income of $\leq 30000$ NTD (66.3\%). In terms of disease characteristics, most respondents had no other comorbidities $(54.3 \%)$, and their mean duration of RDs was 8.4 years ( \pm 5.1 years).

\section{Reliability}

The internal reliability of the C-ASES, as assessed by Cronbach's $\alpha$, was $0.82,0.84$ and 0.89 for the pain, other symptoms and physical function subscales, respectively, and 0.91 for the total scale (table 2). The item-total correlations ranged from 0.48 to 0.73 . In the test-retest investigation, 4 of the 100 individuals had missing data at the second measurement; thus, calculation of test-retest reliability was performed on data provided by 96 subjects via ICC and the Bland-Altman analysis. The ICC was 0.89 for the C-ASES total scores, 0.85 for the pain subscale, 0.88 
Table 1 Demographic and clinical characteristics of enrolled subjects

\begin{tabular}{|c|c|c|c|c|}
\hline \multirow[b]{2}{*}{ Variable } & \multicolumn{2}{|c|}{ All subjects $(n=258)$} & \multicolumn{2}{|c|}{$\begin{array}{l}\text { Subjects of convince sample } \\
(n=96)\end{array}$} \\
\hline & Mean \pm SD & N (\%) & Mean \pm SD & $\mathrm{N}(\%)$ \\
\hline \multicolumn{5}{|c|}{ Demographic characteristics } \\
\hline \multicolumn{5}{|l|}{ Educational level } \\
\hline High ( $\geq$ ninth grade) & & $132(51.2)$ & & $54(56.2)$ \\
\hline Low (<ninth grade) & & $126(48.8)$ & & $46(43.8)$ \\
\hline \multicolumn{5}{|l|}{ Martial status } \\
\hline Married & & $218(84.5)$ & & $81(84.4)$ \\
\hline Single & & $40(15.5)$ & & $15(15.6)$ \\
\hline \multicolumn{5}{|l|}{ Monthly income } \\
\hline$\leq 30000$ NTD & & $171(66.3)$ & & $52(54.2)$ \\
\hline$>30000$ NTD & & $87(33.7)$ & & $44(45.8)$ \\
\hline \multicolumn{5}{|l|}{ Sex } \\
\hline Female & & $192(74.4)$ & & $79(82.3)$ \\
\hline Male & & $66(25.6)$ & & $17(82.3)$ \\
\hline Age (years) & $57.1(14.1)$ & & $53.8(10.9)$ & \\
\hline \multicolumn{5}{|l|}{ Clinical characteristics } \\
\hline \multicolumn{5}{|l|}{ Comorbidity } \\
\hline Yes & & $118(45.7)$ & & $68(70.9)$ \\
\hline No & & $140(54.3)$ & & $28(29.1)$ \\
\hline Disease duration (years) & $8.4(5.1)$ & & & $5.1(4.3)$ \\
\hline
\end{tabular}

NTD, New Taiwan Dollars.

for the other symptoms' subscale and 0.90 for the physical function subscale; all supported the test-retest reliability. In addition, the plot from the Bland-Altman test showed that most of the measured differences between the first and the second measurements approached 0 and fell within the limits of agreement (mean $\pm 1.96 \mathrm{SD}$; figure 1$)$, and the change in the slope of linear regression line did not reach statistical significance $(\beta=0.341 ; \mathrm{p}=0.11)$.

\section{Content validity and face validity}

A panel of clinical rheumatological experts conducted a CVI test. The instrument was sent to four experts, covering two rheumatologists, one nurse with over 5 years of nursing experience in the rheumatologic department and a nursing professor to assess whether each item was relevant and appropriate as an indicator of the SE construct. The panel of experts used a 4-point Likert scale to evaluate the content of the C-ASES for relevance of content, comprehensiveness of measure and clarity of items, while considering the cultural context appropriateness for each item. The CVI for the C-ASES in this survey was 0.91 . In addition, the pre-final C-ASES was examined by interviewing 30 volunteers with RDs and requesting that they make suggestions on any difficult-to-answer items. Finally, no any problematic word was reported after the process.

\section{Concurrent validity}

Table 3 shows the concurrent validity of the C-ASES as assessed by correlation analysis with the TDQ. Significantly moderate correlations were found between TDQ and the pain, physical function and other symptoms subscales, with correlation coefficients of -0.53 to -0.54 and -0.67 , respectively. The negative correlation coefficients indicate that a higher level of depression was related to a lower degree of SE.

\section{Construct validity}

We further applied exploratory factor analysis to confirm the construct validity of the C-ASES. First, the KMO method and Bartlett's test of sphericity were used to assess the suitability of factor analysis. The KMO value was 0.90 , and the statistical significance of Bartlett's test of sphericity $\left(\chi^{2}=3160.34 ; \mathrm{p}<0.001\right)$ indicated a highly compact pattern of inter-item correlations, thus justifying the use of factor analysis. ${ }^{15} 1929 \mathrm{~A}$ principal component analysis with varimax rotations extracted three factors from these 20 items, based on eigenvalues greater than 1.0 and factor loading greater than 0.4; each item loaded onto the 3 established domains as expected and explained 59.78\% of the total variance. Factor 1 appears to reflect a 'physical function' factor based on the content of its 9 items, explaining $30.97 \%$ of the total item variance. 
Table 2 Mean values, SD, correlations and Cronbach's alpha coefficients of items of the C-ASES

\begin{tabular}{|ccccc}
\hline Items & Mean & SD & r & Cronbach's $\boldsymbol{~}$ \\
\hline Pain & & & & 0.82 \\
\hline Q1 & 72.25 & 34.10 & 0.60 & \\
\hline Q2 & 84.69 & 24.53 & 0.68 & \\
\hline Q3 & 79.53 & 30.87 & 0.66 & \\
\hline Q4 & 49.03 & 37.76 & 0.49 & \\
\hline Q5 & 47.25 & 37.82 & 0.48 & \\
\hline Physical function & & & & 0.89 \\
\hline Q1 & 91.36 & 21.77 & 0.54 & \\
\hline Q2 & 72.91 & 33.17 & 0.59 & \\
\hline Q3 & 85.62 & 27.85 & 0.65 & \\
\hline Q4 & 94.22 & 18.07 & 0.60 & \\
\hline Q5 & 94.11 & 18.17 & 0.58 & \\
\hline Q6 & 95.39 & 15.13 & 0.54 & \\
\hline Q7 & 84.46 & 28.28 & 0.55 & \\
\hline Q8 & 92.75 & 21.82 & 0.60 & \\
\hline Q9 & 94.07 & 16.99 & 0.50 & \\
\hline Other symptoms & & & & 0.84 \\
\hline Q1 & 80.50 & 32.08 & 0.51 & \\
\hline Q2 & 80.00 & 31.36 & 0.63 & \\
\hline Q3 & 81.82 & 31.00 & 0.60 & \\
\hline Q4 & 67.13 & 29.51 & 0.54 & \\
\hline Q5 & & & & 0.91 \\
\hline Q6 & & 27.00 & 0.73 & \\
\hline All & & & \\
\hline
\end{tabular}

C-ASES, Chinese version of Arthritis Self-Efficacy Scale.

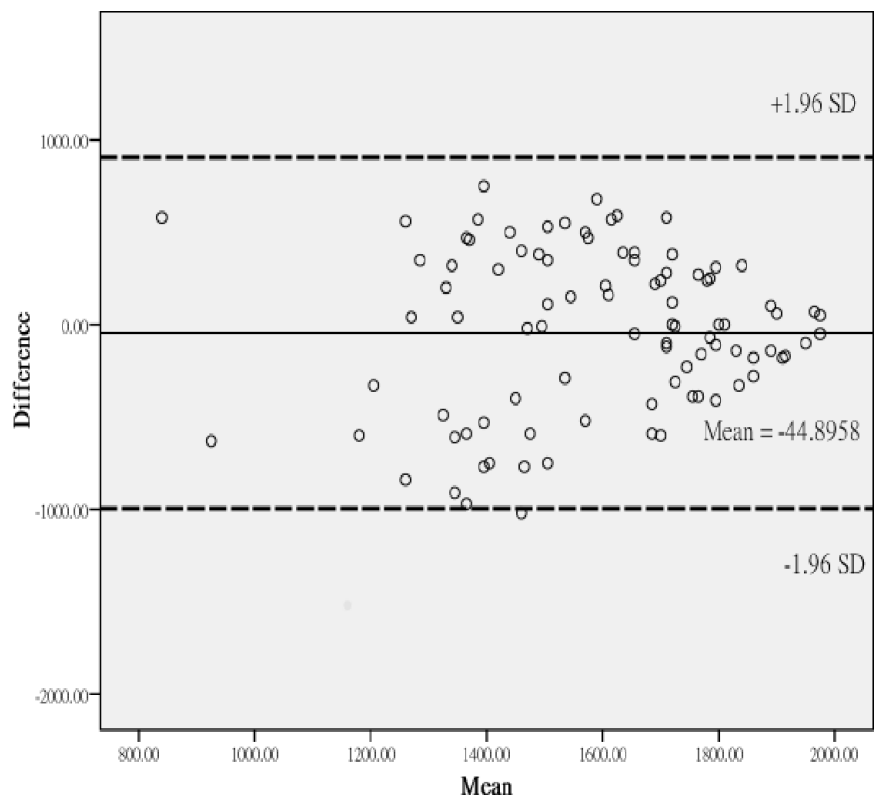

Figure 1 Bland-Altman plot assessing agreement between two measuring points.
Table 3 Bivariate correlations of the C-ASES and the TDQ $(\mathrm{n}=258)$

\begin{tabular}{ll}
\hline ASES & TDQ \\
\hline Physical function & $-0.54^{\star}$ \\
Other symptoms & $-0.67^{\star}$ \\
Pain & $-0.53^{\star}$ \\
Total & $-0.69^{\star}$ \\
\hline
\end{tabular}

${ }^{* *} \mathrm{P}<0.001$

ASES, Arthritis Self-Efficacy Scale; C-ASES, Chinese version of ASES; TDQ, Taiwanese Depression Questionnaire.

Factor 2, which accounted for $16.91 \%$ of the variance, can be labelled 'other symptoms' based on the content of its 6 items. The remaining 5 items explain $11.90 \%$ of the variance and correspond to the third factor 'pain' (table 4).

\section{DISCUSSION \\ Main findings}

Aside from receiving proper medical treatment, a patient's perceived SE is also crucial in improving clinical prognosis. ${ }^{15}$ The timely awareness of SE has been recognised as a first step in instituting appropriate interventions. ${ }^{814}$ To the best of our knowledge, this is the first study to assess the reliability and validity of ASES in Chinese patients with RDs, allowing for future validation of its psychometric properties in people of other cultures.

\section{Comparison of the current study with previous studies \\ Reliability assessment}

Findings from our study indicated that the Cronbach's $\alpha$ of C-ASES total score, the pain, physical function and other symptoms subscales were $0.91,0.82$, 0.89 and 0.84 , respectively, all of which conformed to the typically established criterion that $\alpha$ should be greater than $0.7 .{ }^{24}{ }^{30}$ After an extensive review of the literature, we found only the internal consistency measured by Cronbach's $\alpha$ was reported, ranging from 0.76 to $0.91,{ }^{891416}$ and our estimations did not differ much from those. In addition, in our study, the item-to-total score correlations ranged from 0.48 to 0.73 , indicating that individual items were consistent with the scale's assessed construct. A major contribution of the present study is to examine the reproducibility as determined by ICC as well as the Bland-Altmann analysis. ICC values for the total scale and the subscales were all greater than 0.80 . Moreover, the plot from Bland-Altmann analysis revealed that the discrepancies between two measuring points were random and almost all fell within the $95 \%$ limits of agreement. It can, therefore, be concluded that the C-ASES has satisfactory reproducibility among Chinese patients with RDs. Of particular importance was that the majority of previous studies merely 
Table 4 Exploratory factor analysis of C-ASES

\begin{tabular}{|c|c|c|c|}
\hline Items & Eigenvalue & $\begin{array}{l}\text { Variance } \\
\text { explained (\%) }\end{array}$ & $\begin{array}{l}\text { Factor } \\
\text { loading }\end{array}$ \\
\hline Factor 1: physical function & 8.19 & 30.97 & \\
\hline $\begin{array}{l}\text { Q13. Are you confident that you are able to enter or get out of the } \\
\text { passenger seat of a car without assistance from other people or } \\
\text { assistive devices? }\end{array}$ & & & 0.80 \\
\hline $\begin{array}{l}\text { Q9. Are you confident that you can fasten and unfasten three medium- } \\
\text { sized buttons in a row within } 12 \mathrm{~s} \text { ? }\end{array}$ & & & 0.79 \\
\hline
\end{tabular}

Q10. Are you confident that you can use a knife and fork to cut two

roughly bite-sized pieces of meat within $8 \mathrm{~s}$ ?

Q14. Are you confident that you can put on a long-sleeve or short-

sleeve shirt that opens at the front within $8 \mathrm{~s}$ (without fastening the

buttons)?

Q6. Are you confident that you can walk 100 feet on level ground within

20s?

Q11. Are you confident that you can easily open or close an outdoor

faucet?

Q8. Are you confident that you can get out of an armless chair quickly

0.66

without using your hands at all?

Q12. Are you confident that you can use both hands to scratch your

upper back?

Q7. Are you confident that you can climb up 10 stairs in $7 \mathrm{~s}$ ?

0.54

Factor 2: other symptoms

3.09

0.76

Q17. How confident are you that you can do something to help you

break out of a depressed mood?

Q19. How confident are you that you can adapt to your joint symptoms

and complete the tasks you wish to finish?

Q20. How confident are you that you can deal with the sense of

0.74

frustration that comes from your joint disease?

Q16. Are you confident that, after an adjustment in your level of activity,

your joint disease will not worsen?

Q15. Are you confident that you can control your fatigue?

0.61

Q18. Compared with other people with joint disease, how confident are

0.57

you that you can deal with your joint pain as you go about your daily

activities?

\section{Factor 3: pain}

2.20

11.89

Q5. How certain are you that you can make a large reduction in your arthritis pain by using methods other than taking extra medication?

Q4. How certain are you that you can make a small-to-moderate

reduction in your arthritis pain by using methods other than taking extra medication?

Q3. How confident are you that you can tolerate your joint pain enough so that it will not affect your sleep?

Q1. How confident are you that you can significantly reduce your own

pain?

Q2. How confident are you that you can engage in most of your daily activities?

Total

C-ASES, Chinese version of Arthritis Self-Efficacy Scale.

relied on Pearson product moment correlations to determine test-retest reliability, ${ }^{91416}$ thus potentially risking violation of the independence assumption across variables and attenuating the psychometric soundness of the ASES when applied across different cultures. 


\section{Validity assessment}

The overall validity of C-ASES was supported by assessments of its content, construct and concurrent validities. First, four expert raters evaluated the clinical appropriateness of the scale's 20 items. None of the 20 items had a CVI lower than 0.80 and the overall CVI score was above 0.90 , thus demonstrating good agreement among the four experts as to the viability of the instrument to measure SE. Earlier studies seldom reported CVI values of the ASES among patients with arthritis, ${ }^{9} 1417$ making a direct comparison to our findings impossible. However, our findings did meet the psychometric standard proposed by Polit and Beck in which the item-level CVI should exceed $0.78 .^{31}$

Another commonly used method to assess a questionnaire's validity is through factor analysis. ${ }^{19}$ Before performing the exploratory factor analysis, the KMO was calculated to be 0.90 , which indicated that the sample was large enough for a satisfactory factor analysis. ${ }^{19} 19$ Therefore, we inferred that the size of the recruited sample was abundant. Echoing earlier reports ${ }^{15}{ }^{16}$ the exploratory factor analysis extracted three factors which corresponded to the original ASES subscales. None of the items demonstrated observable psychometric weaknesses, and nearly $60 \%$ of the total scale variance was explained by the three-factor solution. Additionally, examination of the concurrent validity revealed that higher levels of depression were associated with lower SE, concurring with former findings. ${ }^{25}$ Taken together, these findings are consistent with earlier studies reporting that SE is linked to, and predictive of, meaningful physical and psychological health outcomes among arthritic patients. ${ }^{89} 13$

\section{Study strengths and weaknesses}

This study provides evidence for semantic, construct and conceptual equivalence of the C-ASES, but some limitations are worth noting as additional directions for future research. First, all participants were drawn from a single hospital in southern Taiwan and, therefore, may not be representative of other Chinese-speaking populations. Future studies should recruit larger samples or adopt random sampling procedures to assess the applicability of the C-ASES to all ethnically Chinese populations. Nonetheless, we considered the sample size needed to ensure adequate statistical power before initiating this preliminary study, so the sample size appears to be satisfactory for the purposes of the current study. Second, due to time constraints, the researchers were unable to obtain data pertaining to the depression measure over time, thus limiting the ability to examine the predictive validity of C-ASES. Future research should use longitudinal measurement of additional clinical prognostics, in addition to depression, to investigate the utility of $\mathrm{SE}$ as a predictor of the indicators of RA-triggered distress. Third, our findings focused mainly on the empirically driven subscales of C-ASES, as derived by exploratory factor analysis rather than confirmatory factor analysis. Nevertheless, the exploratory factor analysis is more useful to investigate the original factor structure of the instrument. ${ }^{27}$ Despite these methodological concerns, to the best of our knowledge, this was the first study to determine the validity and reliability of ASES among Chinese patients with RDs. The examination of the psychometric properties of the scale supported the usefulness of ASES as a culturally appropriate instrument for the measurement of SE.

In conclusion, the results of this study, obtained by rigorous procedures, confirm that the Chinese-language version of the ASES possesses acceptable content, concurrent and construct validities, as well as internal consistency and temporal stability. This instrument may be useful for assessment of SE and helpful for healthcare providers in tailoring appropriate interventions for patients with RDs. Given that lower levels of SE have been found, in prior studies, to be related to poorer clinical outcomes, targeted screening and detection of influencing factors of SE may, therefore, represent an important strategy for detecting and improving clinical manifestations among patients with RDs.

\section{Author affiliations}

${ }^{1}$ Department of Medical Research, Dalin Tzu Chi Hospital, Buddhist Tzu Chi Medical Foundation, 2 Minsheng Road, Dalin Township, Chiayi 62247, Taiwan

${ }^{2}$ Department of Nursing, Tzu Chi University of Science and Technology, 880 ChienKuo, Hualien, Taiwan

${ }^{3}$ Department of Environmental and Occupational Health, College of Medicine, National Cheng Kung University, Tainan, Taiwan

${ }^{4}$ Division of Allergy, Immunology and Rheumatology, Dalin Tzu Chi Hospital, Buddhist Tzu Chi Medical Foundation, 2 Minsheng Road, Dalin Township, Chiayi 62247, Taiwan

${ }^{5}$ School of Medicine, Tzu Chi University, Hualien, Taiwan

${ }^{6}$ Rehabilitation Counseling Program, Portland State University, Portland, Oregon, USA

${ }^{7}$ Department of Nursing, Dalin Tzu Chi Hospital, Buddhist Tzu Chi Medical

Foundation, 2 Minsheng Road, Dalin Township, Chiayi 62247, Taiwan

${ }^{8}$ Department of Occupational and Environmental Medicine, National Cheng Kung University Hospital, Tainan, Taiwan

${ }^{9}$ Occupational Safety, Health, and Medicine Research Center, National Cheng Kung University, Tainan, Taiwan

Correction notice This article has been corrected since it was first published. Name for the author Ning-Sheng Lai has been corrected.

Acknowledgements We thank the co-investigators of this project and the patients who responded to our survey. We are also grateful for Dr Lorig's contribution during the backward translation of Chinese version of Arthritis Self-Efficacy Scale. T-YT, HL and $\mathrm{M}-\mathrm{CL}$ contributed equally to this work. T-YT and H-RG were both corresponding authors.

Contributors All the authors approved the contents of the submitted article. Conceived and designed the experiments: M-CL, HL and T-YT. Analysed the data: H-RG and T-YT. Contributed reagents/materials/analysis tools: T-YT, HL, M-CL and $\mathrm{N}-\mathrm{SL}$. Wrote the paper: T-YT, HL, M-CL and H-RG. Final approval of manuscript: M-CL, HL, T-YT, M-CL, N-SL and H-RG.

Funding This study was supported by a research grant by Dalin Tzu Chi Hospital (Grant No.: DTCRD107-E-14).

Competing interests None declared.

Patient consent for publication Not required.

Ethics approval This study was approved by the Research Ethics Committee of Dalin Tzu Chi Hospital (no.: B10603007).

Provenance and peer review Not commissioned; externally peer reviewed.

Data availability statement Data are available upon reasonable request. The data used and/or analysed during the current study are available from the corresponding authors on academic research and reasonable request.

Supplemental material This content has been supplied by the author(s). It has not been vetted by BMJ Publishing Group Limited (BMJ) and may not have been peer-reviewed. Any opinions or recommendations discussed are solely those 
of the author(s) and are not endorsed by BMJ. BMJ disclaims all liability and responsibility arising from any reliance placed on the content. Where the content includes any translated material, BMJ does not warrant the accuracy and reliability of the translations (including but not limited to local regulations, clinical guidelines, terminology, drug names and drug dosages), and is not responsible for any error and/or omissions arising from translation and adaptation or otherwise.

Open access This is an open access article distributed in accordance with the Creative Commons Attribution Non Commercial (CC BY-NC 4.0) license, which permits others to distribute, remix, adapt, build upon this work non-commercially, and license their derivative works on different terms, provided the original work is properly cited, appropriate credit is given, any changes made indicated, and the use is non-commercial. See: http://creativecommons.org/licenses/by-nc/4.0/.

\section{ORCID iDs}

Tzung-Yi Tsai http://orcid.org/0000-0002-9865-7101

Ming-Chi Lu http://orcid.org/0000-0001-9051-0351

How-Ran Guo http://orcid.org/0000-0003-3343-3446

\section{REFERENCES}

1 Barbour KE, Helmick CG, Boring M, et al. Vital Signs: Prevalence of Doctor-Diagnosed Arthritis and Arthritis-Attributable Activity Limitation - United States, 2013-2015. MMWR Morb Mortal Wkly Rep 2017:66:246-53.

2 Centers for Disease Control and Prevention (CDC). National and state medical expenditures and lost earnings attributable to arthritis and other rheumatic conditions--United States, 2003. MMWR Morb Mortal Wkly Rep 2007;56:4-7.

3 National Health Insurance Administration. Receipts, itemized medical expenses, medication Lists. Available: https://www.nhi. gov.tw/english/Content_List.aspx?n=C614E7B87C9EC77C\&topn= 1D1ECC54F86E9050 [Accessed 12 Oct 2020].

4 Gabriel SE, Michaud K. Epidemiological studies in incidence, prevalence, mortality, and comorbidity of the rheumatic diseases. Arthritis Res Ther 2009;11:229.

5 Kendzerska T, Jüni P, King LK, et al. The longitudinal relationship between hand, hip and knee osteoarthritis and cardiovascular events: a population-based cohort study. Osteoarthritis Cartilage 2017;25:1771-80.

6 Lu M-C, Guo H-R, Lin M-C, et al. Bidirectional associations between rheumatoid arthritis and depression: a nationwide longitudinal study. Sci Rep 2016;6:20647.

7 Veronese N, Cereda E, Maggi S, et al. Osteoarthritis and mortality: a prospective cohort study and systematic review with meta-analysis. Semin Arthritis Rheum 2016;46:160-7.

8 Primdahl J, Wagner L, Hørslev-Petersen K. Self-efficacy as an outcome measure and its association with physical disease-related variables in persons with rheumatoid arthritis: a literature review. Musculoskeletal Care 2011;9:125-40.

9 Brady TJ. Measures of self-efficacy: Arthritis Self-Efficacy Scale (ASES), Arthritis Self-Efficacy Scale-8 Item (ASES-8), Children's Arthritis Self-Efficacy Scale (CASE), Chronic Disease Self-Efficacy Scale (CDSES), Parent's Arthritis Self-Efficacy Scale (PASE), an. Arthritis Care Res 2011;63:S473-85.
10 Mueller A, Hartmann M, Mueller K, et al. Validation of the arthritis self-efficacy short-form scale in German fibromyalgia patients. Eur $J$ Pain 2003;7:163-71.

11 Ünsal A, Kasikci Mk. Effect of education on perceived self-efficacy for individuals with arthritis. Int J Caring Sci 2010;3:3-11.

12 Bandura A. Self-efficacy: toward a unifying theory of behavioral change. Psychol Rev 1977:84:191-215.

13 Brus $H$, van de Laar M, Taal E, et al. Determinants of compliance with medication in patients with rheumatoid arthritis: the importance of self-efficacy expectations. Patient Educ Couns 1999;36:57-64.

14 Garratt AM, Løchting I, Smedslund G, et al. Measurement properties of instruments assessing self-efficacy in patients with rheumatic diseases. Rheumatology 2014;53:1161-71.

15 Lorig K, Chastain RL, Ung E, et al. Development and evaluation of a scale to measure perceived self-efficacy in people with arthritis. Arthritis Rheum 1989;32:37-44.

16 Lomi C. Evaluation of a Swedish version of the arthritis self-efficacy scale. Scand J Caring Sci 1992;6:131-8.

17 Brekke M, Hjortdahl P, Kvien TK. Self-efficacy and health status in rheumatoid arthritis: a two-year longitudinal observational study. Rheumatology 2001;40:387-92.

18 Hosseini Moghadam M, Jahanbin I, Nazarinia MA. The effect of educational program on self-efficacy of women with rheumatoid arthritis: a randomized controlled clinical trial. Int J Community Based Nurs Midwifery 2018;6:12-20.

19 Gorsuch RL. Factor analysis. 2nd ed. New York: Lawrence Erlbaum Associates, 1983.

20 Taal E, Riemsma RP, Brus HL, et al. Group education for patients with rheumatoid arthritis. Patient Educ Couns 1993;20:177-87.

21 Gjersing L, Caplehorn JRM, Clausen T. Cross-cultural adaptation of research instruments: language, setting, time and statistical considerations. BMC Med Res Methodol 2010;10:13.

22 Lee Y, Yang MJ, Lai TJ, et al. Development of the Taiwanese depression questionnaire. Chang Gung Med J 2000;23:688-94.

23 Lin M-C, Lu M-C, Livneh $\mathrm{H}$, et al. Factors associated with sexual dysfunction in Taiwanese females with rheumatoid arthritis. BMC Womens Health 2017:17:12.

24 Chiang $\mathrm{H}-\mathrm{H}$, Guo $\mathrm{H}-\mathrm{R}$, Livneh $\mathrm{H}$, et al. Increased risk of progression to dialysis or death in CKD patients with depressive symptoms: a prospective 3-year follow-up cohort study. J Psychosom Res 2015;79:228-32.

25 Wu S-FV, Huang Y-C, Lee M-C, et al. Self-efficacy, self-care behavior, anxiety, and depression in Taiwanese with type 2 diabetes: a crosssectional survey. Nurs Health Sci 2013;15:213-9.

26 Marx RG, Menezes A, Horovitz L, et al. A comparison of two time intervals for test-retest reliability of health status instruments. J Clin Epidemiol 2003;56:730-5.

27 Bland JM, Altman DG. Measuring agreement in method comparison studies. Stat Methods Med Res 1999;8:135-60.

28 Wu S-F. [A two-stage translation and test the validity and reliability of a foreign instrument]. Hu Li Za Zhi 2006;53:65-71.

29 Kaiser HF. An index of factorial simplicity. Psychometrika 1974:39:31-6.

30 Bland JM, Altman DG. Statistics notes: Cronbach's alpha. BMJ 1997;314:572.

31 Polit DF, Beck CT. The content validity index: are you sure you know what's being reported? critique and recommendations. Res Nurs Health 2006;29:489-97. 